\title{
Relationship between Vitamin Intake and Depressive Symptoms in Elderly Japanese Individuals: Differences with Gender and Body Mass Index
}

\author{
Thao Thi Thu Nguyen, Hiromasa Tsujiguchi, Yasuhiro Kambayashi, Akinori Hara, Sakae Miyagi, \\ Yohei Yamada, Haruki Nakamura, Yukari Shimizu, Daisuke Hori, Fumihiko Suzuki, \\ Koichiro Hayashi and Hiroyuki Nakamura * \\ Department of Environmental and Preventive Medicine, Graduate School of Medical Science, \\ Kanazawa University, 13-1 Takara-machi, Kanazawa 920-8640, Japan; toi_fs@yahoo.com (T.T.T.N.); \\ t-hiromasa@med.kanazawa-u.ac.jp (H.T.); ykamba@med.kanazawa-u.ac.jp (Y.K.); ahara@m-kanazawa.jp (A.H.); \\ smiyagi@staff.kanazawa-u.ac.jp (S.M.); yamada503597@gmail.com (Y.Y.); \\ haruki_nakamura_kanazawa@yahoo.co.jp (H.N.); h_zu@me.com (Y.S.); hori_d@mbr.nifty.com (D.H.); \\ fumi@dental.email.ne.jp (F.S.); orihciok1003@gmail.com (K.H.) \\ * Correspondence: hiro-n@po.incl.ne.jp; Tel.: +81-76-265-2218
}

Received: 13 October 2017; Accepted: 30 November 2017; Published: 3 December 2017

\begin{abstract}
Only a few studies have focused on the relationship between vitamin intake and depressive symptoms in Japanese individuals. This cross-sectional study investigated the relationship between vitamin intake and depressive symptoms in 1634 elderly Japanese individuals (65 years and older). The consumption of fifteen vitamins including retinol, a retinol equivalent, beta-carotene equivalent, vitamin $\mathrm{D}$, alpha-tocopherol, vitamin $\mathrm{K}$, vitamin group $\mathrm{B}$, vitamin $\mathrm{C}$, and cryptoxanthine was analyzed using a brief-type self-administered diet history questionnaire (BDHQ). The short version of the Geriatric Depression Scale (GDS) was used to assess depressive symptoms. The prevalence of participants with depressive symptoms was $26.7 \%$. The consumption of all vitamins, except for retinol and vitamin $\mathrm{D}$, was lower among depressed than non-depressed participants. The consumption of vitamins was significantly less in female and overweight participants with depressive symptoms than in elderly participants without depressive symptoms. After adjustments for potential confounders, none of the fifteen vitamins were correlated with depressive symptoms in male or underweight participants. Associations between vitamin deficiencies and depressive symptoms were observed in female and overweight elderly participants. Our findings demonstrated a relationship between vitamin intake and depressive symptoms.
\end{abstract}

Keywords: depression; vitamin; elderly individuals; Japanese

\section{Introduction}

Depression is a major cause of illness in societies worldwide, affecting personal well-being, ability to work, and the use of healthcare resources [1]. The total estimated number of individuals living with depression globally is 322 million [2], with an increase of $18.4 \%$ being observed between 2005 and 2015 [3]. In elderly individuals, depression is considered to be the most frequent cause of emotional suffering and significantly decreases quality of life [4-6]. The prevalence of depression varies, and is higher among woman. Its prevalence in Korean middle-aged and older adults was previously reported to be $4.1 \%$ in men and $11.7 \%$ in women [7]. Another study on an older Chinese population suggested that the risk of depression was 1.98-fold higher in women than in men [8]. A study on 3113 community-dwelling individuals aged 40 years or older in Japan reported more severe depressive symptoms in $4.3 \%$ of male and $6.3 \%$ of female participants [9]. 
Previous studies have also investigated the reciprocal relationship between being overweight, obesity, and depression [10,11]. A cross-sectional study using Korean National Health Examination Survey data showed a relationship between a higher body weight and more severe depressive symptoms [12]. A three-year longitudinal epidemiological design among older adults suggested that body mass index (BMI) was associated with depressive symptoms; however, the effect size was small [13]. In contrast, other studies found a negative or no relationship between depression and being overweight/obese [14,15].

Depression in the elderly is complex and may occur for various reasons and under different conditions. Diet and nutrition, particularly vitamins, influence depression. Mikkelsen et al. have demonstrated in their studies that deficiency in vitamin B such as B1, B3, B6, B9, B12 has been linked to depression $[16,17]$. Previous findings indicated that fat-soluble antioxidant micronutrients, such as vitamin $\mathrm{E}$, protect against neural damage, with a low dietary intake of vitamin $\mathrm{E}$ being related to altered mood and depression $[18,19]$. In other studies, a correlation was found between depression and decreased serum 25-hydroxyvitamin $\mathrm{D}(25(\mathrm{OH}) \mathrm{D})$ levels in older individuals [20,21]. A systematic review and meta- analysis study have indicated an inverse association between serum $25(\mathrm{OH}) \mathrm{D}$ levels and the risk of depression [22]. However, another systematic review study has shown that the evidence linking vitamin D intake to non-clinically depressed in healthy individuals was inconsistent; larger, longer trials suggested that this evidence was not reliable [23]. Studies that have reviewed the association between vitamin A and depression have suggested that retinoic acid, an active form of vitamin A, can cause depression and suicide in some susceptible individuals [24,25]. The study by Oishi J showed that carotene, vitamin $\mathrm{C}$ intakes were associated with lowering depressive symptoms among community-dwelling elderly persons in Japan [26]. Intervention trials have also provided evidence for the effectiveness of vitamin supplements in the treatment of depression [27-29].

Although Japan was listed as a country with a lower prevalence of depression in a cross-sectional study on eighteen high- and low-to-middle-income countries [30], the number of patients with depression has been increasing [31]. Studies on vitamin intake have been conducted among elderly Japanese individuals; however, evidence of a relationship between vitamin intake and depression is limited, particularly in categorized groups. Therefore, we conducted this study on older individuals in order to investigate the relationship between vitamin deficiencies and depressive symptoms with differences in gender and BMI.

\section{Materials and Methods}

\subsection{Study Population}

The present cross-sectional study was based on the Shika Study, which has been conducted in Shika town, Ishikawa prefecture since 2011. The Shika Study included interviews, self-administered questionnaires, and a comprehensive health examination.

Shika town is located in Ishikawa prefecture with a population of 21,061 individuals, of which approximately $40 \%$ are elderly (65 years and older) and $56 \%$ of individuals are older than 40 years of age. We selected four model districts in Shika town for the study including Horimatsu, Higashi-masuho, Tsuchida and Togi with a population in 2016 of 7183 and 2592 households [32]. The invitation letters to participate in the study were sent to all individuals from 40 years of age between January 2015 to January 2016. The study population of the Shika study consisted of 4121 individuals from 40 years of age. Persons were eligible to participate in the study if they were 65 or older and capable of giving informed consent. The present study was conducted on 1634 elderly participants aged 65 years and older (39.65\% of all participants from 40 years of age). Written informed consent was obtained from all participants in the survey. The present study complied with Declaration of Helsinki Guidelines and it was approved by the Ethical Committee at Kanazawa University. The recruitment of subjects is shown in Figure 1. 


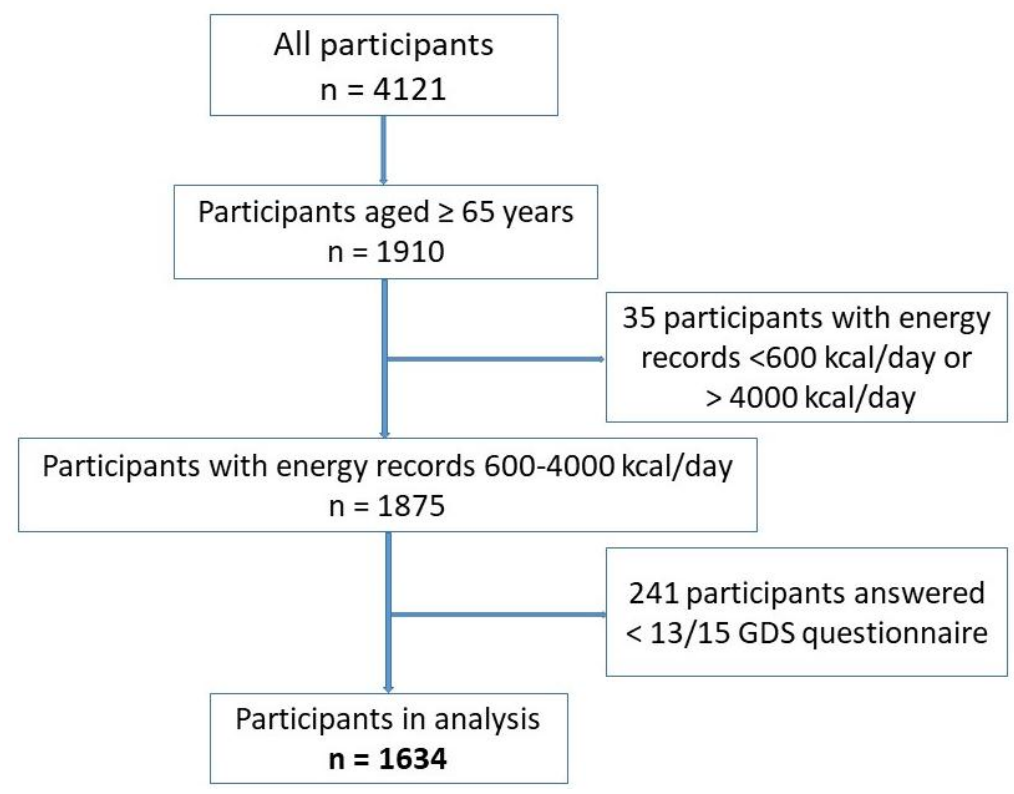

Figure 1. Participant recruitment chart.

\subsection{Depression}

Depressive symptoms were assessed using the short version of the Geriatric Depression Scale (GDS), a self-administered survey consisting of 15 yes/no questions with higher scores indicating higher depressive symptomatology [33]. A review article on the criterion validity of GDS has suggested that the most often a cut-off value of 5 or 6 was used [34]. A Japanese version of the 15-item GDS has been evaluated for validity and reliability in a Japanese population with the recommended optimal cut-off score of 6/7 [35]. In the present study, we used a predefined cut-off point of 6 to define depressive symptoms and non-depressive symptoms; subjects with a GDS score from 6 were categorized as having depressive symptoms, while those with a GDS score of less than 6 were categorized as having non-depressive symptoms (a cut-off score of 6 for the GDS had a sensitivity of 0.973 and specificity of 0.959 ) [36]. We only included participants who answered more than 13 out of the 15 questions in the analysis.

\subsection{Nutrients Assessment}

A brief-type self-administered diet history questionnaire (BDHQ) was used to analyze nutritional intake including the consumption of 15 vitamins and their compounds: retinol, a retinol equivalent, beta-carotene equivalent, vitamin D, alpha-tocopherol, vitamin K, vitamin group B (B1, B2, B3, B5, B6, B9, and B12), vitamin C, and cryptoxanthine. In the present study, we used a validated BDHQ, which was designed to obtain information on each individual's nutritional and food intakes as well as a few markers of dietary behavior during the previous month in order to assess the amount of nutrients habitually consumed from food typically eaten (excluding intake from dietary supplements) [37-39]. The BDHQ dietary intake was based on the consumption frequency of selected food and nonalcoholic beverage items, the daily intake of rice and miso soup, usual cooking methods for fish and meat, and general dietary behavior [38,39]. The estimated vitamin intake (g/day) for 58 food and beverage items, which are most commonly consumed in Japan with some modifications using a food list suggested by the National Health and Nutrition Survey of Japan [40], were calculated using an ad-hoc computer algorithm based on the Japanese standard of food composition table [41] which included weighting factors for the BDHQ [42].

We also adjusted the basic index from the BDHQ, including total energy, protein, lipid, and carbohydrate intakes. We excluded all participants who reported a total energy intake of less than 
$600 \mathrm{kcal} /$ day (half of the required energy for the lowest physical activity category) or more than $4000 \mathrm{kcal} /$ day (1.5 times the energy intake required for the moderate physical activity category) due to under/over-estimations leading to bias in the analysis of other nutrients $[37,40,41]$.

\subsection{Other Variables}

BMI was calculated as current body weight $(\mathrm{kg})$ divided by the square of body height $(\mathrm{m})$. Subjects were grouped into 3 categories: underweight (BMI less than 18.5), normal weight (BMI ranging between 18.5 and 24.99), and overweight (BMI of 25 and higher).

Participants also reported their living status (living alone or with someone), marital status (single, widowed, separated), smoking status (current smoker, had stopped, non-smoker), alcohol drinking, and history of chronic diseases (hypertension, stroke, myocardial infarction, diabetes, hyperlipidemia). In our population, participants who reported a history of stroke and/or myocardial infarction also had hypertension and/or diabetes. Therefore, we focused on hypertension, diabetes, and hyperlipidemia as confounders in the analysis.

\subsection{Statistical Analysis}

Vitamin intake was adjusted for energy using the density method as a percentage of the daily energy intake for energy-containing nutrients. Differences in characteristics and vitamin intake between participants with and without depressive symptoms were assessed using the $x^{2}$ test (categorical variables) and Student's $t$-test (continuous variables).

Confounding variables were gender, age, height, weight, BMI, living conditions, marital status, smoking status, alcohol drinking, and a history of hypertension, diabetes, and hyperlipidemia.

Participants were divided into gender and BMI groups for analyses. The odd ratios (ORs) and 95\% confidence intervals (CIs) of each vitamin for depressive symptoms were calculated by univariate and multivariate logistic regression models with adjustments for potential confounding factors in order to assess the relationship between vitamin intake and depressive symptoms. Data were statistically analyzed using the SPSS software program for MS Windows, version 23.0 (SPSS, Inc., New York, NY, USA). The significance of differences was set at $p<0.05$ for all analyses.

\section{Results}

\subsection{Participant Characteristics According to the Depressive Symptoms Status}

Among 1634 participants $(74.48 \pm 7.43$ years old $), 55.94 \%(n=914)$ were women and $437(26.7 \%)$ exhibited depressive symptoms, of which $26.8 \%$ females (245/914) and $26.6 \%$ males $(192 / 720)$ had depressive symptoms (Supplementary Table S1). Participants with depressive symptoms were significantly older than participants without depressive symptoms. In male participants, those with depressive symptoms had a significantly lower energy intake $(1893.0 \pm 582.1 \mathrm{kcal} /$ day $)$ than participants without depressive symptoms (2058.7 $\pm 644.4 \mathrm{kcal} /$ day); however, they had a significantly higher carbohydrate intake (137.8 \pm 21.8 compared to $133.9 \pm 22.2 \mathrm{~g} / 1000 \mathrm{kcal})$. Similar results were observed in females (Table 1).

Vitamin intake was compared between depressive symptoms and participants without depressive symptoms. The levels of thirteen out of fifteen vitamins were lower in participants with depressive symptoms, except for retinol and vitamin $\mathrm{D}$. The levels of the entire vitamin group B were significantly lower in participants with depressive symptoms (Table 2). 
Table 1. Participant characteristics.

\begin{tabular}{|c|c|c|c|c|c|c|c|}
\hline & & \multicolumn{3}{|c|}{ Male } & \multicolumn{3}{|c|}{ Female } \\
\hline & & $\begin{array}{l}\text { Non-Depressive Symptoms } \\
\qquad(n=528)\end{array}$ & $\begin{array}{c}\text { Depressive Symptoms } \\
\quad(n=192)\end{array}$ & $p 1$ & $\begin{array}{l}\text { Non-Depressive Symptoms } \\
\qquad(n=669)\end{array}$ & $\begin{array}{c}\text { Depressive Symptoms } \\
(n=245)\end{array}$ & $p 2$ \\
\hline \multicolumn{2}{|c|}{ Age (years) (mean \pm SD) } & $73.0 \pm 6.6$ & $75.1 \pm 7.3$ & 0.001 & $74.10 \pm 7.3$ & $78.3 \pm 8.2$ & $<0.001$ \\
\hline \multicolumn{2}{|c|}{ Height $(\mathrm{cm})($ mean \pm SD) } & $163.6 \pm 6.1$ & $162.8 \pm 6.4$ & 0.091 & $150.1 \pm 5.7$ & $148.1 \pm 6.6$ & $<0.001$ \\
\hline \multirow{2}{*}{\multicolumn{2}{|c|}{$\begin{array}{l}\text { Weight }(\mathrm{kg})(\text { mean } \pm \mathrm{SD}) \\
\text { BMI }(\text { mean } \pm \mathrm{SD})\end{array}$}} & $62.5 \pm 9.3$ & $61.6 \pm 10.0$ & 0.224 & $51.6 \pm 8.2$ & $47.9 \pm 8.1$ & $<0.001$ \\
\hline & & $23.3 \pm 2.9$ & $23.3 \pm 3.3$ & 0.707 & $22.9 \pm 3.2$ & $21.8 \pm 3.4$ & $<0.001$ \\
\hline \multirow{3}{*}{ BMI groups } & Underweight (115) & $22(4.2 \%)$ & $10(5.2 \%)$ & \multirow{3}{*}{0.741} & $46(6.9 \%)$ & $37(15.1 \%)$ & \multirow{3}{*}{$<0.001$} \\
\hline & Normal weight (1141) & $371(70.3 \%)$ & $130(67.7 \%)$ & & $466(69.7 \%)$ & $174(71.0 \%)$ & \\
\hline & Overweight (378) & $135(35.6 \%)$ & $52(27.1 \%)$ & & $157(23.5 \%)$ & $34(13.9 \%)$ & \\
\hline Energy & (kcal/day) & $2058.7 \pm 644.4$ & $1893.0 \pm 582.1$ & 0.001 & $1697.7 \pm 544.3$ & $1607.3 \pm 557.0$ & 0.027 \\
\hline Protein & (g/1000 kcal) & $38.1 \pm 7.8$ & $38.3 \pm 8.1$ & 0.744 & $40.9 \pm 8.6$ & $38.8 \pm 7.8$ & 0.001 \\
\hline \multirow{2}{*}{\multicolumn{2}{|c|}{$\begin{array}{l}\text { Lipids (g/1000 kcal) } \\
\text { Carbohydrates (g/1000 kcal) }\end{array}$}} & $26.1 \pm 6.2$ & $26.2 \pm 6.4$ & 0.846 & $28.9 \pm 6.5$ & $27.5 \pm 6.8$ & 0.005 \\
\hline & & $133.9 \pm 22.2$ & $137.8 \pm 21.8$ & 0.036 & $139.9 \pm 20.3$ & $144.7 \pm 19.8$ & 0.001 \\
\hline
\end{tabular}

BMI: body mass index; SD: standard deviation. 
Table 2. Differences in vitamin intake between the groups with and without depressive symptoms.

\begin{tabular}{cccc}
\hline & $\begin{array}{c}\text { Non-Depressive Symptoms } \\
(\boldsymbol{n}=\mathbf{1 1 9 7})\end{array}$ & $\begin{array}{c}\text { Depressive Symptoms } \\
(\boldsymbol{n}=\mathbf{4 3 7 )}\end{array}$ & $\boldsymbol{p}$ \\
\cline { 2 - 4 } Vitamin (g/1000 kcal) & Vitamin Intake (mean $\pm \mathbf{S D})$ & 0.132 \\
Retinol & $218.11 \pm 266.03$ & $197.54 \pm 169.56$ & 0.011 \\
Retinol equivalent & $401.32 \pm 294.33$ & $361.63 \pm 203.53$ & 0.005 \\
Beta-carotene equivalent & $2176.61 \pm 1412.54$ & $1958.11 \pm 1368.00$ & 0.064 \\
Vitamin D & $9.64 \pm 5.75$ & $9.05 \pm 5.53$ & 0.001 \\
Alpha-tocopherol & $4.02 \pm 1.15$ & $3.08 \pm 1.11$ & $<0.001$ \\
Vitamin K & $181.96 \pm 100.73$ & $156.02 \pm 89.51$ & 0.002 \\
Vitamin B1 & $0.42 \pm 0.10$ & $0.41 \pm 0.10$ & 0.007 \\
Vitamin B2 & $0.72 \pm 0.21$ & $0.69 \pm 0.20$ & $<0.001$ \\
Vitamin B3 & $9.48 \pm 2.89$ & $8.80 \pm 2.64$ & 0.002 \\
Vitamin B5 & $3.58 \pm 0.79$ & $3.45 \pm 0.72$ & $<0.001$ \\
Vitamin B6 & $0.73 \pm 0.20$ & $0.68 \pm 0.18$ & $<0.001$ \\
Vitamin B9 & $190.29 \pm 75.91$ & $171.72 \pm 65.14$ & 0.006 \\
Vitamin B12 & $6.35 \pm 3.35$ & $5.84 \pm 3.09$ & $<0.001$ \\
Vitamin C & $65.78 \pm 32.96$ & $58.61 \pm 30.65$ & 0.019 \\
Cryptoxanthine & $165.19 \pm 148.49$ & $145.59 \pm 152.49$ &
\end{tabular}

\subsection{Vitamin Intake in Different Groups of Participants}

In males, only vitamin $\mathrm{K}$ levels were significantly lower in participants with depressive symptoms $(148.69 \pm 83.04 \mathrm{mg} / 1000 \mathrm{kcal})$ than in participants without depressive symptoms $(165.52 \pm 89.82 \mathrm{mg} / 1000 \mathrm{kcal})$. In contrast, among females, the intake of all fifteen vitamins was significantly reduced in participants with depressive symptoms (Table 3).

By stratifying according to BMI, participants with an underweight BMI did not show any significant difference in vitamin intake between the group with depressive symptoms and the group without depressive symptoms. Similar differences were observed in normal BMI and overweight BMI participants. In normal BMI participants, the levels of nine out of fifteen vitamins and compounds were significantly lower in participants with depressive symptoms than in participants without depressive symptoms, including the beta-carotene equivalent, alpha-tocopherol, vitamin $\mathrm{K}$, vitamin B1, vitamin B3, vitamin B6, vitamin B9, vitamin B5, and vitamin C. A number of differences were noted in fourteen vitamins and compounds (except for cryptoxanthine), such as a lower intake in overweight participants with depressive symptoms. The intake of the entire vitamin group B was higher in participants without depressive symptoms than in participants with depressive symptoms (Table 4). 
Table 3. Differences in vitamin intake between the groups with and without depressive symptoms by gender.

\begin{tabular}{|c|c|c|c|c|c|c|}
\hline \multirow{3}{*}{ Vitamin (g/1000 kcal) } & \multicolumn{3}{|c|}{ Male $(n=720)$} & \multicolumn{3}{|c|}{ Female $(n=914)$} \\
\hline & $\begin{array}{c}\text { Non-Depressive Symptoms } \\
(n=528,73.3 \%)\end{array}$ & $\begin{array}{c}\text { Depressive Symptoms } \\
\quad(n=192,26.7 \%)\end{array}$ & \multirow[t]{2}{*}{$p 1$} & $\begin{array}{c}\text { Non-Depressive Symptoms } \\
\quad(n=669,73.2 \%)\end{array}$ & $\begin{array}{c}\text { Depressive Symptoms } \\
\quad(n=245,26.8 \%)\end{array}$ & \multirow[t]{2}{*}{$p 2$} \\
\hline & \multicolumn{2}{|c|}{ Vitamin Intake (mean \pm SD) } & & \multicolumn{2}{|c|}{ Vitamin Intake (mean \pm SD) } & \\
\hline Retinol & $237.11 \pm 361.04$ & $223.85 \pm 221.95$ & 0.633 & $203.11 \pm 152.85$ & $176.92 \pm 108.81$ & 0.004 \\
\hline Retinol equivalent & $394.48 \pm 377.24$ & $368.73 \pm 242.38$ & 0.378 & $406.72 \pm 206.79$ & $357.85 \pm 167.28$ & $<0.001$ \\
\hline Beta-carotene equivalent & $1866.66 \pm 1232.40$ & $1715.04 \pm 1181.41$ & 0.140 & $2421.24 \pm 1496.10$ & $2148.59 \pm 1472.59$ & 0.014 \\
\hline Vitamin D & $9.10 \pm 5.37$ & $9.35 \pm 5.63$ & 0.590 & $10.06 \pm 6.01$ & $8.82 \pm 5.46$ & 0.005 \\
\hline Alpha-tocopherol & $3.69 \pm 1.04$ & $3.65 \pm 1.04$ & 0.609 & $4.28 \pm 1.17$ & $3.93 \pm 1.16$ & $<0.001$ \\
\hline Vitamin K & $165.52 \pm 89.82$ & $148.69 \pm 83.04$ & 0.024 & $194.94 \pm 106.87$ & $161.76 \pm 94.01$ & $<0.001$ \\
\hline Vitamin B1 & $0.39 \pm 0.09$ & $0.39 \pm 0.09$ & 0.578 & $0.45 \pm 0.10$ & $0.42 \pm 0.10$ & 0.001 \\
\hline Vitamin B2 & $0.68 \pm 0.20$ & $0.68 \pm 0.20$ & 0.969 & $0.74 \pm 0.21$ & $0.69 \pm 0.20$ & $<0.001$ \\
\hline Vitamin B3 & $9.20 \pm 2.78$ & $8.82 \pm 2.53$ & 0.095 & $9.70 \pm 2.95$ & $8.77 \pm 2.73$ & $<0.001$ \\
\hline Vitamin B5 & $3.39 \pm 0.73$ & $3.39 \pm 0.72$ & 0.933 & $3.74 \pm 0.81$ & $3.50 \pm 0.72$ & $<0.001$ \\
\hline Vitamin B6 & $0.70 \pm 0.18$ & $0.68 \pm 0.18$ & 0.155 & $0.75 \pm 0.21$ & $0.69 \pm 0.19$ & $<0.001$ \\
\hline Vitamin B9 & $178.17 \pm 70.28$ & $168.11 \pm 62.62$ & 0.081 & $199.87 \pm 78.83$ & $174.56 \pm 67.03$ & $<0.001$ \\
\hline Vitamin B12 & $6.22 \pm 3.32$ & $6.12 \pm 3.31$ & 0.708 & $6.45 \pm 3.37$ & $5.62 \pm 2.91$ & 0.001 \\
\hline Vitamin C & $57.45 \pm 29.89$ & $55.11 \pm 32.27$ & 0.363 & $72.34 \pm 33.79$ & $61.36 \pm 29.09$ & $<0.001$ \\
\hline Cryptoxanthine & $132.53 \pm 124.92$ & $142.65 \pm 176.63$ & 0.394 & $190.97 \pm 160.18$ & $147.89 \pm 130.84$ & $<0.001$ \\
\hline
\end{tabular}

SD: standard deviation. 
Table 4. Differences in vitamin intake between the groups with and without depressive symptoms by BMI.

\begin{tabular}{|c|c|c|c|c|c|c|c|c|c|}
\hline \multirow{3}{*}{$\begin{array}{c}\text { Vitamin } \\
\text { (g/1000 kcal) }\end{array}$} & Und & rweight & \multirow{3}{*}{$p 1$} & \multicolumn{3}{|c|}{ Normal Weight } & \multicolumn{3}{|c|}{ Overweight } \\
\hline & $\begin{array}{l}\text { Non-Depressive Symptoms } \\
\qquad(n=68)\end{array}$ & $\begin{array}{l}\text { Depressive Symptoms } \\
\qquad(n=47)\end{array}$ & & $\begin{array}{l}\text { Non-Depressive Symptoms } \\
\qquad(n=837)\end{array}$ & $\begin{array}{c}\text { Depressive Symptoms } \\
\quad(n=304)\end{array}$ & \multirow[t]{2}{*}{$p^{2}$} & $\begin{array}{l}\text { Non-Depressive Symptoms } \\
\qquad(n=292)\end{array}$ & $\begin{array}{c}\text { Depressive Symptoms } \\
\quad(n=86)\end{array}$ & \multirow[t]{2}{*}{$p 3$} \\
\hline & \multicolumn{2}{|c|}{ Vitamin Intake (mean \pm SD) } & & \multicolumn{2}{|c|}{ Vitamin Intake (mean \pm SD) } & & \multicolumn{2}{|c|}{ Vitamin Intake (mean \pm SD) } & \\
\hline Retinol & $210.08 \pm 151.47$ & $193.65 \pm 156.16$ & 0.573 & $218.76 \pm 294.58$ & $204.49 \pm 183.80$ & 0.429 & $218.09 \pm 190.09$ & $175.06 \pm 114.67$ & 0.010 \\
\hline Retinol equivalent & $388.40 \pm 194.04$ & $386.42 \pm 199.02$ & 0.958 & $405.21 \pm 318.96$ & $374.21 \pm 215.49$ & 0.117 & $393.19 \pm 234.44$ & $308.70 \pm 147.56$ & $<0.001$ \\
\hline Beta-carotene equivalent & $2117.37 \pm 1544.73$ & $2286.89 \pm 1619.20$ & 0.572 & $2215.12 \pm 1395.75$ & $2013.97 \pm 1405.64$ & 0.033 & $2080.02 \pm 1428.29$ & $1580.93 \pm 960.37$ & $<0.001$ \\
\hline Vitamin D & $9.29 \pm 5.09$ & $10.14 \pm 5.54$ & 0.398 & $9.66 \pm 5.88$ & $9.10 \pm 5.46$ & 0.150 & $9.67 \pm 5.55$ & $8.27 \pm 5.75$ & 0.043 \\
\hline Alpha-tocopherol & $4.05 \pm 1.09$ & $4.02 \pm 1.28$ & 0.897 & $4.02 \pm 1.17$ & $3.83 \pm 1.09$ & 0.016 & $4.02 \pm 1.11$ & $3.58 \pm 1.08$ & 0.001 \\
\hline Vitamin K & $180.55 \pm 98.0$ & $153.55 \pm 86.23$ & 0.130 & $183.08 \pm 100.47$ & $162.02 \pm 92.38$ & 0.0 & $179.10 \pm 102.37$ & $136.16 \pm 78.27$ & $<0.001$ \\
\hline Vitamin B1 & $0.42 \pm 0.10$ & $0.43 \pm 0.11$ & & $0.42 \pm 0.10$ & $0.41 \pm 0.09$ & 0.0 & $042 \pm 0.10$ & $0.38 \pm 0.09$ & 0.001 \\
\hline Vitamin B2 & $0.72 \pm 0.20$ & $0.70 \pm 0.18$ & 0.600 & $0.72 \pm 0.21$ & $0.69 \pm 0.21$ & 0.1 & $0.72 \pm 0.22$ & $0.65 \pm 0.19$ & 0.005 \\
\hline Vitamin B3 & $9.17 \pm 2.63$ & $8.81 \pm 2.79$ & 0.488 & $9.49 \pm 2.91$ & $8.90 \pm 2.55$ & 0.0 & $9.55 \pm 2.89$ & $8.41 \pm 2.88$ & 0.001 \\
\hline Vitamin B5 & $3.62 \pm 0.76$ & $3.56 \pm 0.66$ & 0.7 & $3.58 \pm 0.79$ & $3.48 \pm 0.72$ & 0.0 & $3.59 \pm 0.81$ & $3.31 \pm .073$ & 0.003 \\
\hline Vitamin B6 & $0.72 \pm 0.20$ & $0.71 \pm 0.20$ & 0.829 & $0.73 \pm 0.20$ & $0.69 \pm 0.18$ & 0.0 & $0.73 \pm 0.19$ & $0.64 \pm 0.19$ & $<0.001$ \\
\hline Vitamin B9 & $185.01 \pm 69.89$ & $174.95 \pm 69.57$ & 0.449 & $191.74 \pm 76.54$ & $176.09 \pm 66.01$ & 0.001 & $187.39 \pm 75.58$ & $154.52 \pm 56.90$ & $<0.001$ \\
\hline Vitamin B12 & $6.10 \pm 3.01$ & $6.08 \pm 3.37$ & 0.966 & $6.33 \pm 3.39$ & $5.92 \pm 3.06$ & 0.065 & $6.48 \pm 3.33$ & $5.44 \pm 3.09$ & 0.010 \\
\hline Vitamin C & $63.48 \pm 29.69$ & $60.49 \pm 35.16$ & 0.623 & $66.30 \pm 33.55$ & $60.32 \pm 3.17$ & 0.005 & $64.81 \pm 32.01$ & $51.51 \pm 24.98$ & $<0.001$ \\
\hline Cryptoxanthine & $158.82 \pm 124.14$ & $148.58 \pm 162.53$ & 0.703 & $164.99 \pm 149.82$ & $146.52 \pm 153.02$ & 0.067 & $167.25 \pm 150.27$ & $140.65 \pm 146.56$ & 0.148 \\
\hline
\end{tabular}




\subsection{Influence of Gender and BMI on the Relationship between Vitamin Intake and Depressive Symptoms}

Vitamin K, vitamin C, vitamin B1, vitamin B5, vitamin B6, and vitamin B9 deficiencies correlated with depressive symptoms after adjustments for all covariates (Supplementary Table S2). We investigated whether a similar relationship existed between vitamin intake levels and depressive symptoms with differences in gender or BMI. No correlations were observed between vitamin intake and depressive symptoms in underweight participants (Supplementary Table S3) and males (Table 5). In contrast, in females, all vitamins, except for vitamin A1, the beta-carotene equivalent, vitamin $\mathrm{D}$, and vitamin B3, showed a negative correlation with depressive symptoms. Deficiencies in vitamin group B showed the strongest correlation with an increase in the prevalence of depressive symptoms (Table 5).

Table 5. Odds ratios (95\% CI) for depressive symptom outcomes according to vitamin intake stratified by gender*.

\begin{tabular}{|c|c|c|c|c|c|c|}
\hline Vitamin (g/1000 kcal) & \multicolumn{3}{|c|}{ Male } & \multicolumn{3}{|c|}{ Female } \\
\hline Retinol & 0.000 & $1.000(0.999-1.000)$ & 0.779 & -0.001 & 0.999 (0.997-1.000) & 0.098 \\
\hline Beta-carotene equivalent & 0.000 & $1.000(1.000-1.000)$ & 0.065 & 0.000 & $1.000(1.000-1.000)$ & 0.024 \\
\hline Vitamin D & 0.037 & $1.038(0.998-1.080)$ & 0.063 & -0.032 & $0.969(0.935-1.004)$ & 0.080 \\
\hline Alpha-tocopherol & 0.014 & $1.014(0.830-1.239)$ & 0.889 & -0.239 & $0.788(0.645-0.962)$ & 0.020 \\
\hline Vitamin B1 & -0.585 & $0.557(0.066-4.729)$ & 0.592 & -2.148 & $0.117(0.014-0.949)$ & 0.044 \\
\hline Vitamin B2 & -0.045 & $0.956(0.345-2.649)$ & 0.931 & -1.183 & $0.306(0.102-0.921)$ & 0.035 \\
\hline Vitamin B3 & -0.019 & $0.981(0.902-1.067)$ & 0.652 & -0.082 & $0.921(0.847-1.002)$ & 0.055 \\
\hline Vitamin B5 & -0.013 & $0.987(0.754-1.291)$ & 0.923 & -0.388 & $0.678(0.511-0.901)$ & 0.007 \\
\hline Vitamin B6 & -0.323 & $0.724(0.213-2.458)$ & 0.605 & -1.656 & $0.191(0.064-0.570)$ & 0.003 \\
\hline Vitamin B9 & -0.003 & 0.997 (0.995-1.000) & 0.073 & -0.005 & $0.995(0.993-0.998)$ & 0.000 \\
\hline Vitamin B12 & 0.029 & $1.030(0.964-1.099)$ & 0.383 & -0.079 & $0.924(0.862-0.989)$ & 0.023 \\
\hline Vitamin C & -0.004 & $0.996(0.990-1.003)$ & 0.245 & -0.010 & $0.990(0.985-0.996)$ & 0.001 \\
\hline Cryptoxanthine & 0.000 & $1.000(0.999-1.002)$ & 0.542 & -0.002 & $0.998(0.997-0.999)$ & 0.003 \\
\hline
\end{tabular}

* Adjusted for Age, Height, Weight, BMI, Living status, Marital status, Drinking alcohol, Smoking status, Energy, Carbohydrates, Hypertension, Diabetes, and Hyperlipidemia. B: beta coefficient; OR: Odd ratio, CI: confidence interval.

We clarified the relationship between vitamin intake levels and depressive symptoms in females with different BMIs. In an analysis of overweight participants, a correlation was observed between vitamin intake and depressive symptoms for the retinol equivalent, vitamin $\mathrm{D}$, vitamin $\mathrm{K}$, vitamin B2, vitamin B5, and vitamin B9. No correlations were noted between vitamin intake and depressive symptoms in underweight females (Table 6). Furthermore, we did not find any significant association between depressive symptoms and vitamin intake in normal weight and overweight males (Supplementary Table S4) 
Table 6. Odds ratios $(95 \% \mathrm{CI})$ for depressive symptom outcomes according to vitamin intake stratified by BMI in females *

\begin{tabular}{|c|c|c|c|c|c|c|c|c|c|}
\hline \multirow{2}{*}{$\begin{array}{c}\text { Vitamin } \\
\text { (g/1000 kcal) }\end{array}$} & \multirow{2}{*}{ B } & Underweight & \multirow{2}{*}{$p$} & \multirow{2}{*}{ B } & Normal Weight & \multirow{2}{*}{$p$} & \multirow{2}{*}{ B } & Overweight & \multirow{2}{*}{$p$} \\
\hline & & OR $(95 \% \mathrm{CI})$ & & & OR $(95 \% \mathrm{CI})$ & & & OR $(95 \% \mathrm{CI})$ & \\
\hline Retinol & 0.000 & $1.000(0.994-1.007)$ & 0.892 & -0.002 & $0.998(0.997-1.000)$ & 0.134 & -0.005 & $0.995(0.990-1.000)$ & 0.061 \\
\hline Retinol equivalent & 0.000 & $1.000(0.996-1.004)$ & 0.991 & -0.002 & $0.998(0.997-1.000)$ & 0.012 & -0.004 & $0.996(0.992-0.999)$ & 0.022 \\
\hline $\begin{array}{c}\text { Beta-carotene } \\
\text { equivalent }\end{array}$ & 0.000 & $1.000(1.000-1.000)$ & 0.926 & 0.000 & $1.000(1.000-1.000)$ & 0.039 & 0.000 & $1.000(0.999-1.000)$ & 0.201 \\
\hline Vitamin D & 0.080 & $1.083(0.934-1.257)$ & 0.290 & -0.029 & $0.971(0.933-1.011)$ & 0.153 & -0.133 & $0.876(0.771-0.995)$ & 0.042 \\
\hline Alpha-tocopherol & -0.299 & $0.742(0.369-1.492)$ & 0.402 & -0.253 & $0.777(0.613-0.983)$ & 0.036 & -0.185 & $0.831(0.449-1.538)$ & 0.555 \\
\hline Vitamin K & -0.007 & $0.993(0.985-1.000)$ & 0.056 & -0.002 & $0.998(0.996-1.000)$ & 0.043 & -0.009 & $0.992(0.985-0.998)$ & 0.015 \\
\hline Vitamin B1 & -1.998 & $0.136(0.00-229.57)$ & 0.598 & -2.552 & $0.078(0.006-0.963)$ & 0.047 & -4.167 & $0.016(0.000-6.160)$ & 0.172 \\
\hline Vitamin B2 & -1.668 & $0.189(0.01-30.431)$ & 0.520 & -0.850 & $0.427(0.119-1.535)$ & 0.192 & -3.852 & $0.021(0.001-0.479)$ & 0.015 \\
\hline Vitamin B3 & 0.095 & $1.099(0.804-1.503)$ & 0.553 & -0.082 & $0.921(0.834-1.017)$ & 0.104 & -0.136 & $0.873(0.692-1.102)$ & 0.254 \\
\hline Vitamin B5 & -0.648 & $0.523(0.169-1.619)$ & 0.261 & -0.309 & $0.734(0.524-1.029)$ & 0.072 & -0.950 & $0.387(0.181-0.826)$ & 0.014 \\
\hline Vitamin B6 & -1.056 & $0.348(0.008-15.34)$ & 0.585 & -1.737 & $0.176(0.047-0.657)$ & 0.010 & -2.692 & $0.068(0.003-1.433)$ & 0.084 \\
\hline Vitamin B9 & -0.004 & $0.996(0.985-1.006)$ & 0.419 & -0.004 & $0.996(0.993-0.999)$ & 0.005 & -0.010 & $0.990(0.982-0.999)$ & 0.023 \\
\hline Vitamin B12 & 0.108 & $1.114(0.842-1.473)$ & 0.450 & -0.077 & $0.926(0.855-1.003)$ & 0.060 & -0.196 & $0.822(0.672-1.006)$ & 0.057 \\
\hline Vitamin C & -0.008 & $0.992(0.972-1.012)$ & 0.426 & -0.010 & $0.991(0.984-0.997)$ & 0.004 & -0.014 & $0.986(0.969-1.003)$ & 0.112 \\
\hline Cryptoxanthin & -0.002 & $0.998(0.994-1.002)$ & 0.361 & -0.002 & $0.998(0.996-0.999)$ & 0.007 & -0.001 & $0.999(0.996-1.002)$ & 0.588 \\
\hline
\end{tabular}

* Adjusted for Age, Height, Weight, BMI, Living status, Marital status, Drinking alcohol, Smoking status, Energy, Carbohydrates, Hypertension, Diabetes, and Hyperlipidemia. B: beta coefficient, OR: odd ratio, CI: confidence interval. 


\section{Discussion}

The results of the present cross-sectional study suggested that among elderly Japanese individuals, vitamin intake was lower in participants with depressive symptoms than in participants without depressive symptoms, particularly in females and those who were overweight. Moreover, vitamin intake was negatively associated with depressive symptoms in these groups. To the best of our knowledge, this is the first study to demonstrate an inverse relationship between vitamin intake and depressive symptoms in elderly Japanese female and overweight participants.

The present results confirmed previous findings showing that vitamin intake is inversely associated with the diagnosis of depression. Vitamin group B plays a major role in health, with deficiencies being linked to symptoms of psychiatric disorders and depression as well as altered memory function, cognitive impairment, and dementia $[16,43]$. In the present study, participants with depressive symptoms had lower levels of all vitamin group B, and a logistic regression with adjustments for confounders confirmed the negative relationship between depressive symptoms and the levels of vitamin B1, B5, B6, and B9 in all participants. Beside vitamin group B, the effects of other vitamins related to depression have been revealed. The effects of vitamin $\mathrm{K}$ on the central nervous system have mainly been studied under in vitro conditions. Based on its antioxidant actions [44], vitamin $\mathrm{K}$ has been shown to protect neurons and oligodendrocytes from oxidative damage [45] and the naphthoquinone ring was found to be responsible for this neuroprotective action [46]. Our results revealed a negative relationship between vitamin $\mathrm{K}$ and depressive symptoms, which is consistent with the findings of an experimental study on rats [47]. However, a study using Center for Epidemiologic Studies Depression Scale (CES-DS) for Children on school children aged 6-9 years old in Valencia, Spain, identified a positive relationship between children with depressive symptoms and vitamin $\mathrm{K}$ [48]. The difference observed between these findings may be due to the difference in target subjects (children vs elderly population) and depression scale (CES-DS vs. GDS). On the other hand, findings on the effects of vitamin $C$ on depression in healthy individuals and patients with various medical conditions are contradictory. Randomized double-blind, placebo-controlled trial studies showed that vitamin C elevated the mood [49] and decreased the severity of depression [50]. Nevertheless, supplementary vitamin $C$ did not decrease the depression score in type 2 diabetic patients [51]. Our results suggest that a deficiency in vitamin $C$ increases the prevalence of depressive symptoms. Furthermore, the results showing a relationship between a decrease in cryptoxanthine levels and depression were consistent with previous findings [52].

Among female subjects, a relationship between depressive symptoms and vitamin intake was observed for six out of the seven vitamins in vitamin group B (except for vitamin B3), vitamin C, vitamin $\mathrm{K}$, the retinol equivalent, alpha-tocopherol, and cryptoxanthine. Among male participants, although vitamin $\mathrm{K}$ intake was significantly lower in the participants with depressive symptoms than in participants without depressive symptoms, by regression analysis, no association was observed between vitamin intake and depressive symptoms. The present results are consistent with the findings of previous studies that investigated the relationship between vitamin group B and the prevalence of depression $[27,53,54]$. Previous findings indicated that a higher dietary intake of vitamin B6 among women and vitamin B12 among men is protective against late-life depression among generally healthy seniors living in the community [53]. A cross-sectional study among men aged between 40 and 60 years old in Finland did not find any relationships between cobalamin, pyridoxine, riboflavin, and depressive symptoms, but did for folate after adjustments [55]. In the present study, no significant differences were observed in vitamin D intake between groups with or without depressive symptoms, which was in contrast to previous findings [21,56]. The relationship between serum $25(\mathrm{OH}) \mathrm{D}$ concentrations and the prevalence of depression has been suggested to be stronger among women than men. An integrative review has suggested a significant association between mood disorders and low vitamin D levels, indicating that some biochemical mechanism may exist between the two variables [57]. In addition, a study on older adult males in Europe revealed an inverse relationship between 25(OH)D levels and depression that was largely independent of several lifestyle and health factors. Nevertheless, our 
results were similar to those obtained in studies conducted in China and the US [58,59]. Moreover, even though vitamin $\mathrm{D}$ has been suggested to protect against depression, this was not evident among older adults [60]. However, most of these studies focused on general subjects, limiting appropriate comparisons with our results to different genders.

Since depression and being overweight/obese are now very common, they have become popular areas of study. Although the relationship between obesity and depression has been examined, their causal relationship has not yet been elucidated. The present study focused on the relationship between vitamin intake and depressive symptoms in different BMI groups and the results obtained suggested that depressive symptoms were negatively associated with vitamin intake in the normal weight group and overweight group, but not in the underweight group. The present results showed a negative correlation between the retinol equivalent, vitamin $\mathrm{K}$, vitamin B6, vitamin B9, and vitamin $\mathrm{C}$ and depressive symptoms in higher BMI groups. BMI is considered to be an important biomarker of malnutrition [61], and is closely related to depression in older adults [62]. Moreover, being overweight/obese has been proven to correlate with depression $[63,64]$. One of the reasons for this may be a diet imbalance. The diet consumed by Japanese individuals is typically characterized by a high intake of rice, soya products, fish, seaweed, and green tea and a low intake of animal fat and soft drinks [65]. In cross-sectional studies on the general population, a healthy Japanese dietary pattern, characterized by a higher intake of vegetables, fruit, mushrooms, soya products, vitamin B9, and vitamin C, was associated with a lower prevalence of depressive symptoms [66,67]. The present results showed a higher carbohydrate intake in males and females with depression than in non-depressed participants; therefore, we cannot completely eliminate the risk associated with high carbohydrate foods, which may lead to obesity, with low vitamin levels. Moreover, although consistent misreporting across all types of foods may not have influenced dietary energy density estimates, previous studies indicated that overweight individuals may selectively under-report their intake of fatty or sugary foods, which may result in the underestimation of dietary energy density as well as other nutrients.

\section{Limitations}

Several limitations of the present study warrant mention. The cross-sectional design of the present study does not permit the assessment of causality owing to the uncertain temporality of the association. All self-reported dietary assessment methods are subject to random and systematic measurement errors and the misreporting of dietary intakes, particularly by overweight/obese individuals, is also a limitation associated with self-reported dietary assessment methods [68]. Besides that, using an estimated questionnaire to determine nutrient intake may not give accurate results in terms of the value of nutrients taken from blood serum. Therefore, the nutrient intake values in this study may not be an exact representative value for the whole population. In addition, although we adjusted for various potential confounding variables, residual confounding variables such as physical activity, economic income, a history of drug use (particularly for participants with chronic diseases), a history of related diseases such as dementia (diagnosed by physicians), and a history of using vitamin supplements cannot be ruled out.

\section{Conclusions}

The results of the present observational study indicate a relationship between depressive symptoms and vitamin deficiencies, particularly in female and overweight elderly participants. However, these participants may have had reduced dietary intakes of B vitamins, vitamin K, vitamin C, and others due to an imbalanced daily diet. Further studies using serum vitamins in blood in different gender and BMI groups need to be conducted in order to confirm the results of the present study.

Supplementary Materials: The following are available online at www.mdpi.com/2072-6643/9/12/1319/s1, Table S1: Participant characteristics stratified by depressive symptoms and non-depressive symptoms groups. Table S2: Odds ratios $(95 \% \mathrm{CI})$ for depressive symptom outcomes according to vitamin intake in all participants. Table S3: Odds ratios ( $95 \%$ CI) for depressive symptom outcomes according to vitamin intake stratified by BMI in 
all participants. Table S4: Odds ratios $(95 \% \mathrm{CI})$ for depressive symptom outcomes according to vitamin intake stratified by BMI in males.

Acknowledgments: We thank all the participants for their cooperation. This study was supported by Grant-in-Aid for Scientific Research (B) by the Ministry of Education, Culture, Sport, Science and Technology (MEXT), number $15 \mathrm{H} 04783$.

Author Contributions: Study concept and design: Hiroyuki Nakamura and Thao Thi Thu Nguyen. Acquisition of data: Thao Thi Thu Nguyen, Hiromasa Tsujiguchi and Yasuhiro Kambayashi. Analysis and interpretation of data: Thao Thi Thu Nguyen, Yasuhiro Kambayashi, Hiromasa Tsujiguchi, Akinori Hara, Sakae Miyagi, Yukari Shimizu, Yohei Yamada, Haruki Nakamura, Daisuke Hori, Fumihiko Suzuki and Koichiro Hayashi. Drafting and critical revision of the manuscript: Thao Thi Thu Nguyen, Hiromasa Tsujiguchi, Yasuhiro Kambayashi and Hiroyuki Nakamura. All authors read and approved the final manuscript.

Conflicts of Interest: The authors declare no conflict of interest.

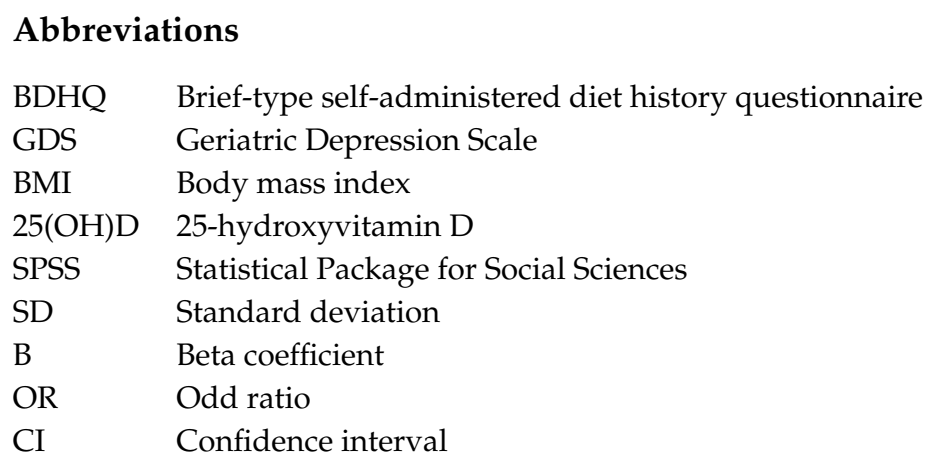

\section{References}

1. Wittchen, H.U.; Jacobi, F.; Rehm, J.; Gustavsson, A.; Svensson, M.; Jönsson, B.; Olesen, J.; Allgulander, C.; Alonso, J.; Faravelli, C.; et al. The size and burden of mental disorders and other disorders of the brain in Europe 2010. Eur. Neuropsychopharmacol. 2011, 21, 655-679. [CrossRef] [PubMed]

2. Depression and Other Common Mental Disorders Global Health Estimates. Available online: http:/ /apps.who. int/iris/bitstream/10665/254610/1/WHO-MSD-MER-2017.2-eng.pdf?ua=1 (accessed on 22 May 2017).

3. GBD 2015 Disease and Injury Incidence and Prevalence Collaborators. Global, regional, and national incidence, prevalence, and years lived with disability for 310 diseases and injuries, 1990-2015: A systematic analysis for the Global Burden of Disease Study 2015. Lancet 2016, 388, 1545-1602. [CrossRef]

4. Doraiswamy, P.M.; Khan, Z.M.; Donahue, R.M.J.; Richard, N.E. The spectrum of quality-of-life impairments in recurrent geriatric depression. J. Gerontol. A Biol. Sci. Med. Sci. 2002, 57, M134-M137. [CrossRef] [PubMed]

5. Blazer, D.G. Depression in Late Life: Review and Commentary. J. Gerontol. Ser. A Biol. Sci. Med. Sci. 2003, 58, M249-M265. [CrossRef]

6. Blazer, D.; Burchett, B.; Service, C.; George, L.K. The Association of Age and Depression Among the Elderly: An Epidemiologic Exploration. J. Gerontol. 1991, 46, M210-M215. [CrossRef] [PubMed]

7. Ra, J.S.; Kim, H.S. Sex-based Association between Depression and Metabolic Syndrome in Korean Middle-aged and Older Adults. Osong Public Heal. Res. Perspect. 2017, 8, 130-137. [CrossRef] [PubMed]

8. Guo, J.; Guan, L.; Fang, L.; Liu, C.; Fu, M.; He, H.; Wang, X. Depression among Chinese older adults: A perspective from Hukou and health inequities. J. Affect. Disord. 2017, 223, 115-120. [CrossRef] [PubMed]

9. Sekita, A.; Arima, H.; Ninomiya, T.; Ohara, T.; Doi, Y.; Hirakawa, Y.; Fukuhara, M.; Hata, J.; Yonemoto, K.; $\mathrm{Ga}, \mathrm{Y}$; et al. Elevated depressive symptoms in metabolic syndrome in a general population of Japanese men: A cross-sectional study. BMC Public Health 2013, 13, 862. [CrossRef] [PubMed]

10. Pereira-Miranda, E.; Costa, P.R.F.; Queiroz, V.A.O.; Pereira-Santos, M.; Santana, M.L.P. Overweight and Obesity Associated with Higher Depression Prevalence in Adults: A Systematic Review and Meta-Analysis. J. Am. Coll. Nutr. 2017, 36, 223-233. [CrossRef] [PubMed]

11. Luppino, F.S.; de Wit, L.M.; Bouvy, P.F.; Stijnen, T.; Cuijpers, P.; Penninx, B.W.; Zitman, F.G. Overweight, Obesity, and Depression A Systematic Review and Meta-analysis of Longitudinal Studies. Arch. Gen. Psychiatry 2010, 67, 220. [CrossRef] [PubMed] 
12. Brewis, A.A.; Han, S.Y.; SturtzSreetharan, C.L. Weight, gender, and depressive symptoms in South Korea. Am. J. Hum. Biol. 2017, e22972. [CrossRef] [PubMed]

13. Sachs-Ericsson, N.; Burns, A.B.; Gordon, K.H.; Eckel, L.A.; Wonderlich, S.A.; Crosby, R.D.; Blazer, D.G. Body Mass Index and Depressive Symptoms in Older Adults: The Moderating Roles of Race, Sex, and Socioeconomic Status. Am. J. Geriatr Psychiatry 2007, 15, 815-825. [CrossRef] [PubMed]

14. Ho, R.C.M.; Niti, M.; Kua, E.H.; Ng, T.-P. Body mass index, waist circumference, waist-hip ratio and depressive symptoms in Chinese elderly: A population-based study. Int. J. Geriatr. Psychiatry 2008, 23, 401-408. [CrossRef] [PubMed]

15. John, U.; Meyer, C.; Rumpf, H.-J.; Hapke, U. Relationships of psychiatric disorders with overweight and obesity in an adult general population. Obes Res. 2005, 13, 101-109. [CrossRef] [PubMed]

16. Mikkelsen, K.; Stojanovska, L.; Apostolopoulos, V. The Effects of Vitamin B in Depression. Curr. Med. Chem. 2016, 23, 4317-4337. [CrossRef] [PubMed]

17. Mikkelsen, K.; Stojanovska, L.; Prakash, M.; Apostolopoulos, V. The effects of vitamin B on the immune/cytokine network and their involvement in depression. Maturitas 2017, 96, 58-71. [CrossRef] [PubMed]

18. Banikazemi, Z.; Mokhber, N.; Safarian, M.; Mazidi, M.; Mirzaei, H.; Esmaily, H.; Azarpazhooh, M.R.; Ghafouri-Taleghani, F.; Ghayour-Mobarhan, M.; Ferns, G.A. Dietary vitamin E and fat intake are related to Beck's depression score. Clin. Nutr. ESPEN 2015, 10, e61-e65. [CrossRef] [PubMed]

19. Maes, M.; De Vos, N.; Pioli, R.; Demedts, P.; Wauters, A.; Neels, H.; Christophe, A. Lower serum vitamin E concentrations in major depression: Another marker of lowered antioxidant defenses in that illness. J. Affect. Disord. 2000, 58, 241-246. [CrossRef]

20. Bertone-Johnson, E.R.; Powers, S.I.; Spangler, L.; Brunner, R.L.; Michael, Y.L.; Larson, J.C.; Millen, A.E.; Bueche, M.N.; Salmoirago-Blotcher, E.; Liu, S.; et al. Vitamin D intake from foods and supplements and depressive symptoms in a diverse population of older women. Am. J. Clin. Nutr. 2011, 94, 1104-1112. [CrossRef] [PubMed]

21. Hoogendijk, W.J.G.; Lips, P.; Dik, M.G.; Deeg, D.J.; Beekman, A.T.; Penninx, B.W. Depression Is Associated With Decreased 25-Hydroxyvitamin D and Increased Parathyroid Hormone Levels in Older Adults. Arch. Gen. Psychiatry 2008, 65, 508. [CrossRef] [PubMed]

22. Ju, S.-Y.; Lee, Y.-J.; Jeong, S.-N. Serum 25-hydroxyvitamin D levels and the risk of depression: A systematic review and meta-analysis. J. Nutr. Health Aging 2013, 17, 447-455. [CrossRef] [PubMed]

23. Morgan, A.J.; Jorm, A.F. Self-help interventions for depressive disorders and depressive symptoms: A systematic review. Ann. Gen. Psychiatry 2008, 7, 13. [CrossRef] [PubMed]

24. Bremner, J.D.; Shearer, K.D.; McCaffery, P.J. Retinoic acid and affective disorders: The evidence for an association. J. Clin. Psychiatry 2012, 73, 37-50. [CrossRef] [PubMed]

25. Ludot, M.; Mouchabac, S.; Ferreri, F. Inter-relationships between isotretinoin treatment and psychiatric disorders: Depression, bipolar disorder, anxiety, psychosis and suicide risks. World J. Psychiatry 2015, 5, 222-227. [CrossRef] [PubMed]

26. Oishi, J.; Doi, H.; Kawakami, N. Nutrition and depressive symptoms in community-dwelling elderly persons in Japan. Acta Medica Okayama 2009, 63, 9-17. [CrossRef] [PubMed]

27. Lee, H.K.; Kim, S.Y.; Sok, S.R. Effects of Multivitamin Supplements on Cognitive Function, Serum Homocysteine Level, and Depression of Korean Older Adults With Mild Cognitive Impairment in Care Facilities. J. Nurs. Scholarsh. 2016, 48, 223-231. [CrossRef] [PubMed]

28. Sparling, T.M.; Henschke, N.; Nesbitt, R.C.; Gabrysch, S. The role of diet and nutritional supplementation in perinatal depression: A systematic review. Matern. Child. Nutr. 2017, 13. [CrossRef] [PubMed]

29. Coppen, A.; Bolander-Gouaille, C. Treatment of depression: Time to consider folic acid and vitamin B12. J. Psychopharmacol. 2005, 19, 59-65. [CrossRef] [PubMed]

30. Bromet, E.; Andrade, L.H.; Hwang, I.; Sampson, N.A.; Alonso, J.; de Girolamo, G.; de Graaf, R.; Demyttenaere, K.; Hu, C.; Iwata, N.; et al. Cross-national epidemiology of DSM-IV major depressive episode. BMC Med. 2011, 9, 90. [CrossRef] [PubMed]

31. Patient Survey I Ministry of Health, Labor and Welfare. Available online: http://www.mhlw.go.jp/toukei/ list/10-20.html (accessed on 14 August 2017).

32. Shikatown Population. Available online: http://www.town.shika.ishikawa.jp/jyuumin/shika_town_pop/ shika_population.html (accessed on 30 August 2017). 
33. Burke, W.J.; Roccaforte, W.H.; Wengel, S.P. The Short Form of the Geriatric Depression Scale: A Comparison With the 30-Item Form. Top. Geriatr. 1991, 4, 173-178. [CrossRef]

34. Wancata, J.; Alexandrowicz, R.; Marquart, B.; Weiss, M.; Friedrich, F. The criterion validity of the Geriatric Depression Scale: A systematic review. Acta Psychiatr. Scand. 2006, 114, 398-410. [CrossRef] [PubMed]

35. Sugishita, K.; Sugishita, M.; Hemmi, I.; Asada, T.; Tanigawa, T. A Validity and Reliability Study of the Japanese Version of the Geriatric Depression Scale 15 (GDS-15-J). Clin. Gerontol. 2017, 40, 233-240. [CrossRef] [PubMed]

36. Schreiner, A.S.; Hayakawa, H.; Morimoto, T.; Kakuma, T. Screening for late life depression: Cut-off scores for the Geriatric Depression Scale and the Cornell Scale for Depression in Dementia among Japanese subjects. Int. J. Geriatr Psychiatry 2003, 18, 498-505. [CrossRef] [PubMed]

37. Okubo, H.; Sasaki, S.; Rafamantanantsoa, H.H.; Ishikawa-Takata, K.; Okazaki, H.; Tabata, I. Validation of self-reported energy intake by a self-administered diet history questionnaire using the doubly labeled water method in 140 Japanese adults. Eur. J. Clin Nutr. 2008, 62, 1343-1350. [CrossRef] [PubMed]

38. Kobayashi, S.; Murakami, K.; Sasaki, S.; Okubo, H.; Hirota, N.; Notsu, A.; Fukui, M.; Date, C. Comparison of relative validity of food group intakes estimated by comprehensive and brief-type self-administered diet history questionnaires against $16 \mathrm{~d}$ dietary records in Japanese adults. Public Health Nutr. 2011, 14, 1200-1211. [CrossRef] [PubMed]

39. Kobayashi, S.; Honda, S.; Murakami, K.; Sasaki, S.; Okubo, H.; Hirota, N.; Notsu, A.; Fukui, M.; Date, C. Both comprehensive and brief self-administered diet history questionnaires satisfactorily rank nutrient intakes in Japanese adults. J. Epidemiol. 2012, 22, 151-159. [CrossRef] [PubMed]

40. Ministry of Health, Labour and Welfare of Japan. National Health and Nutrition Survey 2013. Available online: http://ghdx.healthdata.org/record/japan-national-health-and-nutrition-survey-2013 (accessed on 25 August 2017).

41. Standard Tables of Food Composition in Japan-2015-(Seventh Revised Version). Ministry of Education, Culture, Sports, Science and Technology-Japan. 2015. Available online: http:/ /www.mext.go.jp/en/policy/ science_technology / policy/title01/detail01/1374030.htm (accessed on 25 August 2017).

42. Satoshi, S.; Ryoko, Y.; Keiko, A. Self-administered diet history questionnaire developed for health education: A relative validation of the test-version by comparison with 3-day diet record in women. J. Epidemiol. 1998, 8, 203-215. [CrossRef]

43. Mikkelsen, K.; Stojanovska, L.; Tangalakis, K.; Bosevski, M.; Apostolopoulos, V. Cognitive decline: A vitamin B perspective. Maturitas. 2016, 93, 108-113. [CrossRef] [PubMed]

44. Westhofen, P.; Watzka, M.; Marinova, M.; Hass, M.; Kirfel, G.; Müller, J.; Bevans, C.G.; Müller, C.R.; Oldenburg, J. Human vitamin K 2,3-epoxide reductase complex subunit 1-like 1 (VKORC1L1) mediates vitamin K-dependent intracellular antioxidant function. J. Biol Chem. 2011, 286, 15085-15094. [CrossRef] [PubMed]

45. Li, J.; Lin, J.C.; Wang, H.; Peterson, J.W.; Furie, B.C.; Furie, B.; Booth, S.L.; Volpe, J.J.; Rosenberg, P.A. Novel Role of Vitamin K in Preventing Oxidative Injury to Developing Oligodendrocytes and Neurons. J. Neurosci. 2003, 23, 5816-5826. [PubMed]

46. Josey, B.J.; Inks, E.S.; Wen, X.; Chou, C.J. Structure-activity relationship study of vitamin k derivatives yields highly potent neuroprotective agents. J. Med. Chem. 2013, 56, 1007-1022. [CrossRef] [PubMed]

47. Gancheva, S.M.; Zhelyazkova-Savova, M.D. Vitamin K2 Improves Anxiety and Depression but not Cognition in Rats with Metabolic Syndrome: A Role of Blood Glucose? Folia Medica I Folia Medica 2016, 5858, 264-272. [CrossRef] [PubMed]

48. Rubio-López, N.; Morales-Suárez-Varela, M.; Pico, Y.; Livianos-Aldana, L.; Llopis-González, A. Nutrient Intake and Depression Symptoms in Spanish Children: The ANIVA Study. Int. J. Environ. Res. Public Health 2016, 13, 352. [CrossRef] [PubMed]

49. Brody, S. High-dose ascorbic acid increases intercourse frequency and improves mood: A randomized controlled clinical trial. Biol. Psychiatry 2002, 52, 371-374. [CrossRef]

50. Khajehnasiri, F.; Mortazavi, S.B.; Allameh, A.; Akhondzadeh, S. Effect of omega-3 and ascorbic acid on inflammation markers in depressed shift workers in Shahid Tondgoyan Oil Refinery, Iran: A randomized double-blind placebo-controlled study. J. Clin. Biochem. Nutr. 2013, 53, 36-40. [CrossRef] [PubMed] 
51. Mazloom, Z.; Ekramzadeh, M.; Hejazi, N. Efficacy of Supplementary Vitamins C and E on Anxiety, Depression and Stress in Type 2 Diabetic Patients: A Randomized, Single-blind, Placebo-controlled Trial. Pak. J. Biol Sci. 2013, 16, 1597-1600. [CrossRef] [PubMed]

52. Payne, M.E.; Steck, S.E.; George, R.R.; Steffens, D.C. Fruit, Vegetable, and Antioxidant Intakes Are Lower in Older Adults with Depression. J. Acad. Nutr. Diet. 2012, 112, 2022-2027. [CrossRef] [PubMed]

53. Gougeon, L.; Payette, H.; Morais, J.A.; Gaudreau, P.; Shatenstein, B.; Gray-Donald, K. Intakes of folate, vitamin B6 and B12 and risk of depression in community-dwelling older adults: The Quebec Longitudinal Study on Nutrition and Aging. Eur. J. Clin Nutr. 2016, 70, 380-385. [CrossRef] [PubMed]

54. Hodge, A.; Almeida, O.P.; English, D.R.; Giles, G.G.; Flicker, L. Patterns of dietary intake and psychological distress in older Australians: Benefits not just from a Mediterranean diet. Int. Psychogeriatr. 2013, 25, 456-466. [CrossRef] [PubMed]

55. Tolmunen, T.; Voutilainen, S.; Hintikka, J.; Rissanen, T.; Tanskanen, A.; Viinamäki, H.; Kaplan, G.A.; Salonen, J.T. Dietary folate and depressive symptoms are associated in middle-aged Finnish men. J. Nutr. 2003, 133, 3233-3236. [PubMed]

56. Wang, Y.; Yang, H.; Meng, P.; Han, Y. Association between low serum 25-hydroxyvitamin D and depression in a large sample of Chinese patients with type 2 diabetes mellitus. J. Affect. Disord. 2016. [CrossRef] [PubMed]

57. Murphy, P.; Wagner, C. Vitamin D and Mood Disorders Among Women: An Integrative Review. J. Midwifery Womens Health 2008, 53, 440-446. [CrossRef] [PubMed]

58. Pan, A.; Lu, L.; Franco, O.H.; Yu, Z.; Li, H.; Lin, X. Association between depressive symptoms and 25-hydroxyvitamin D in middle-aged and elderly Chinese. J. Affect. Disord. 2009, 118, 240-243. [CrossRef] [PubMed]

59. Zhao, G.; Ford, E.S.; Li, C.; Balluz, L.S. No associations between serum concentrations of 25-hydroxyvitamin D and parathyroid hormone and depression among US adults. Br. J. Nutr. 2010, 104, 1696-1702. [CrossRef] [PubMed]

60. Jääskeläinen, T.; Knekt, P.; Suvisaari, J.; Männistö, S.; Partonen, T.; Sääksjärvi, K.; Kaartinen, N.E.; Kanerva, N.; Lindfors, O. Higher serum 25-hydroxyvitamin D concentrations are related to a reduced risk of depression. Br. J. Nutr. 2017. [CrossRef] [PubMed]

61. Zhang, Z.; Pereira, S.; Luo, M.; Matheson, E. Evaluation of Blood Biomarkers Associated with Risk of Malnutrition in Older Adults: A Systematic Review and Meta-Analysis. Nutrients 2017, 9, 829. [CrossRef] [PubMed]

62. Smoliner, C.; Norman, K.; Wagner, K.-H.; Hartig, W.; Lochs, H.; Pirlich, M. Malnutrition and depression in the institutionalised elderly. Br. J. Nutr. 2017. [CrossRef] [PubMed]

63. Stunkard, A.J.; Faith, M.S.; Allison, K.C. Depression and obesity. Biol. Psychiatry 2003, 54, 330-337. [CrossRef]

64. Faith, M.S.; Matz, P.E.; Jorge, M.A. Obesity-depression associations in the population. J. Psychosom. Res. 2002, 53, 935-942. [CrossRef]

65. Ministry of Health, Labour and Welfare Japan. The National Health and Nutrition Survey in Japan, 2012. 2014. Available online: http:/ / www.mhlw.go.jp/bunya/kenkou/eiyou/h24-houkoku.html (accessed on 25 August 2017).

66. Nanri, A.; Kimura, Y.; Matsushita, Y.; Ohta, M.; Sato, M.; Mishima, N.; Sasaki, S.; Mizoue, T. Dietary patterns and depressive symptoms among Japanese men and women. Eur. J. Clin Nutr. 2010, 64, 832-839. [CrossRef] [PubMed]

67. Miki, T.; Kochi, T.; Kuwahara, K.; Eguchi, M.; Kurotani, K.; Tsuruoka, H.; Ito, R.; Kabe, I.; Kawakami, N.; Mizoue, T.; Nanri, A. Dietary patterns derived by reduced rank regression (RRR) and depressive symptoms in Japanese employees: The Furukawa nutrition and health study. Psychiatry Res. 2015, 229, $214-219$. [CrossRef] [PubMed]

68. Livingstone, M.B.E.; Black, A.E. Markers of the validity of reported energy intake. J. Nutr. 2003, 133, 895S-920S. [PubMed]

(C) 2017 by the authors. Licensee MDPI, Basel, Switzerland. This article is an open access article distributed under the terms and conditions of the Creative Commons Attribution (CC BY) license (http:/ / creativecommons.org/licenses/by/4.0/). 